MATEC Web of Conferences 25, 01014

(2015)

DOI: $10.1051 /$ matec conf/ 20152501014

(c) Owned by the authors, published by EDP Sciences, 2015

\title{
Influence of Complete Coriolis Force on the Dispersion Relation of Ocean Internal-wave in a Background Currents Field
}

\author{
Yongjun Liu* \\ College of Economic and Management, Linyi University, Linyi, Shandong, China \\ Xiaoping Gao \& Tianxia Yu \\ Department of Mechanical and Electrical Engineering, Shandong Water Polytechnic, Rizhao, Shandong, China \\ Jun Yang \\ College of Economic and Management, Linyi University, Linyi, Shandong, China
}

\begin{abstract}
In this thesis, the influence of complete Coriolis force (the model includes both the vertical and horizontal components of Coriolis force) on the dispersion relation of ocean internal-wave under background currents field are studied, it is important to the study of ocean internal waves in density-stratified ocean. We start from the control equation of sea water movement in the background of the non-traditional approximation, and the vertical velocity solution is derived where buoyancy frequency $\mathrm{N}(\mathrm{z})$ gradually varies with the ocean depth $\mathrm{z}$. The results show that the influence of complete Coriolis force on the dispersion relation of ocean internal-wave under background currents field is obvious, and these results provide strong evidence for the understanding of dynamic process of density stratified ocean internal waves.
\end{abstract}

Keywords: Coriolis parameter; internal waves; dispersion relation; non-traditional approximation.

\section{INTRODUCTION}

A number of observational data show both the vertical and horizontal of the ocean internal-wave which is especially not negligible in this study of near inertial internal waves (Ahran ${ }^{[1]}$, Boebel and Zenk ${ }^{[2]}$ and so on), the parameterization problem of the parameters and explain the dispersion relation of ocean internal wave, wave remote sensing information extraction and ocean model of the internal wave. Considering the horizontal component of non-traditional approximation, the recent research for propagation characteristics of linear internal waves in the ocean have aroused wide concern, and many authors such as Kundu and Thomson ${ }^{[3]}$, Gill ${ }^{[4]}$, Kasahara and Gary ${ }^{[5]}$, Gerkema and Shrira ${ }^{[6]}$, Garrett and Munk ${ }^{[7]}$ and so on have studied the internal waves in different forms of approximate solution and numerical solution, but these studies did not give a simple and practical internal waves' dispersion relation expressions without the effect of background flow field on the frequency dispersion relation under non-traditional approximation. Therefore, we provide a normal mode analysis of the linearized Boussinesq model with particular emphasis on internal waves' dispersion relation with the approximate analytical solutions, and the dispersion relation was compared between the non-traditional and traditional approximation in a background flow field. The results show that the influence of complete Coriolis force on the ocean internal wave dispersion

*Corresponding author: 1yj00001@163.com relation in a background currents field is not negligible.

\section{DISPERSION RELATION OF A BOUSSINESQ APPROXIMATION WITH ROTATION}

\subsection{Basic Equations}

It is assumed that the fluid is irrotational and incompressible for continuous density stratification of seawater, Boussinesq and the complete plane approximation (which is also known as a non-traditional approximation) in the linear internal waves of the governing equations (Gerkema and Shrira ${ }^{[6]}$ and reference Phillips ${ }^{[1]}$ ) and the horizontal uniform steady currents field $\overline{\boldsymbol{U}}=\left\{U_{0}(z), V_{0}(z), 0\right\}$

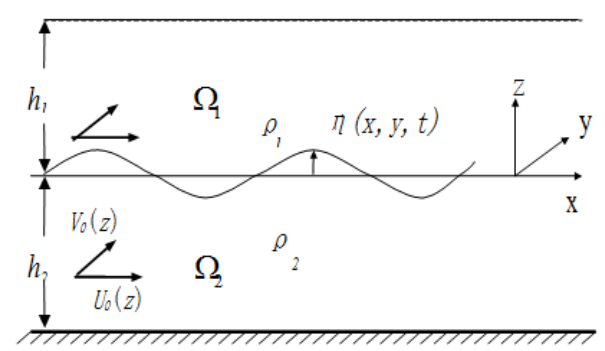

Figure 1. Internal-wave motion diagram in a background current field

This is an Open Access article distributed under the terms of the Creative Commons Attribution License 4.0, which permits unrestricted use, distribution, and reproduction in any medium, provided the original work is properly cited. 


\section{MATEC Web of Conferences}

$\frac{\partial u}{\partial t}+w \frac{\partial U_{0}}{\partial z}+U_{0} \frac{\partial u}{\partial x}+V_{0} \frac{\partial u}{\partial y}-f\left(v+V_{0}\right)+\tilde{f} w=-\frac{\partial p}{\partial x}(1)$

$\frac{\partial v}{\partial t}+w \frac{\partial V_{0}}{\partial z}+U_{0} \frac{\partial v}{\partial x}+V_{0} \frac{\partial v}{\partial y}+f\left(u+U_{0}\right)=-\frac{\partial p}{\partial y}$

$\frac{\partial w}{\partial t}+U_{0} \frac{\partial w}{\partial x}+V_{0} \frac{\partial w}{\partial y}-\tilde{f}\left(u+U_{0}\right)=-\frac{\partial p}{\partial z}+b$

$\frac{\partial b}{\partial t}+U_{0} \frac{\partial b}{\partial x}+V_{0} \frac{\partial b}{\partial y}+N^{2} w=0$

$\frac{\partial u}{\partial x}+\frac{\partial v}{\partial y}+\frac{\partial w}{\partial z}=0$

Here, the coordinates are selected as shown in Figure 1. $\rho, p, u, v$ and $w$ are respectively the corresponding fluid density, the perturbation pressure and the velocity components for $(x, y, z) ; b$ represents the buoyancy; $f=2 \omega \sin \varphi$ and $\hat{f}=2 \omega \cos \varphi$ are respectively corresponding to the vertical and horizontal components of Coriolis force; $\omega$ is the earth's rotation speed; $\varphi$ represents the latitude; $t$ is the time; $h_{1}$ and $h_{2}$ are only corresponding to the different depth of fluids; under the $f$-plane approximation, $f$ and $\hat{f}$ can be considered as constant and we denote

$\frac{d}{d t}=\frac{\partial}{\partial t}+\left(u+U_{0}\right) \frac{\partial}{\partial x}+\left(v+V_{0}\right) \frac{\partial}{\partial y}+w \frac{\partial}{\partial z}$ as the derivative operator.

Rigid lid approximation is used as the upper surface and the bottom boundary condition of the fluid motion,

$w=0, z=0$,

$w=0, z=-H$.

Equations (1)--(5) and boundary conditions (6)--(7) constitute the basic equations for the motion of the sea water in a horizontal uniform current field.

\subsection{Vertical structure equations}

At first, the derivative of Equation (1) is with respect to the variable $z$, then:

$$
\begin{aligned}
& \left(\frac{\partial^{2} u}{\partial t \partial z}+\frac{\partial U_{0}}{\partial z} \frac{\partial u}{\partial x}+U_{0} \frac{\partial^{2} u}{\partial x \partial z}+\frac{\partial V_{0}}{\partial z} \frac{\partial u}{\partial y}+\right. \\
& \left.V_{0} \frac{\partial^{2} u}{\partial y \partial z}+\frac{\partial w}{\partial z} \frac{\partial U_{0}}{\partial z}+w \frac{\partial^{2} U_{0}}{\partial z^{2}}\right)-f\left(\frac{\partial v}{\partial z}+\frac{\partial V_{0}}{\partial z}\right) \\
& +\tilde{f} \frac{\partial w}{\partial z}=-\frac{\partial^{2} p}{\partial x \partial z}
\end{aligned}
$$

Then the derivative of Equation (3) is with respect to the variable $x$, so:

$\left(\frac{\partial^{2} w}{\partial t \partial x}+U_{0} \frac{\partial^{2} w}{\partial x^{2}}+V_{0} \frac{\partial^{2} w}{\partial x \partial y}\right)-\widetilde{f} \frac{\partial u}{\partial x}=-\frac{\partial^{2} p}{\partial x \partial z}+\frac{\partial b}{\partial x}$

Subtract Equation (8) from Equation (9), we obtain:

$\frac{\partial}{\partial t}\left(\frac{\partial w}{\partial x}-\frac{\partial u}{\partial z}\right)+U_{0} \frac{\partial}{\partial x}\left(\frac{\partial w}{\partial x}-\frac{\partial u}{\partial z}\right)$

$+V_{0} \frac{\partial}{\partial y}\left(\frac{\partial w}{\partial x}-\frac{\partial u}{\partial z}\right)-\left(\frac{\partial U_{0}}{\partial z} \frac{\partial u}{\partial x}+\right.$

$\left.\frac{\partial V_{0}}{\partial z} \frac{\partial u}{\partial y}\right)-\frac{\partial}{\partial z}\left(w \frac{\partial U_{0}}{\partial z}\right)+$

$f \frac{\partial}{\partial z}\left(v+V_{0}\right)-\tilde{f}\left(\frac{\partial w}{\partial z}+\frac{\partial u}{\partial x}\right)=\frac{\partial b}{\partial x}$

Derive Equation (10) with respect to the variable $x$, then:

$\frac{\partial}{\partial t}\left(\frac{\partial^{2} w}{\partial x^{2}}-\frac{\partial^{2} u}{\partial x \partial z}\right)+U_{0} \frac{\partial^{2}}{\partial x^{2}}\left(\frac{\partial w}{\partial x}-\frac{\partial u}{\partial z}\right)$

$+V_{0} \frac{\partial^{2}}{\partial x \partial y}\left(\frac{\partial w}{\partial x}-\frac{\partial u}{\partial z}\right)-\left(\frac{\partial U_{0}}{\partial z} \frac{\partial^{2} u}{\partial x^{2}}+\right.$

$\left.\frac{\partial V_{0}}{\partial z} \frac{\partial^{2} u}{\partial x \partial y}\right)-\frac{\partial^{2}}{\partial x \partial z}\left(w \frac{\partial U_{0}}{\partial z}\right)+$

$f \frac{\partial^{2}}{\partial x \partial z}\left(v+V_{0}\right)-\tilde{f}\left(\frac{\partial^{2} w}{\partial x \partial z}+\frac{\partial^{2} u}{\partial x^{2}}\right)=\frac{\partial^{2} b}{\partial x^{2}}$

And then the derivative operator is used in Equation (11), which can be:

$\frac{\partial^{2}}{\partial t^{2}}\left(\frac{\partial^{2} w}{\partial x^{2}}-\frac{\partial^{2} u}{\partial x \partial z}\right)+\left(u+U_{0}\right) \frac{\partial^{2}}{\partial t \partial x}\left(\frac{\partial^{2} w}{\partial x^{2}}-\frac{\partial^{2} u}{\partial x \partial z}\right)+$

$\left(v+V_{0}\right) \frac{\partial^{2}}{\partial t \partial y}\left(\frac{\partial^{2} w}{\partial x^{2}}-\frac{\partial^{2} u}{\partial x \partial z}\right)+w \frac{\partial^{2}}{\partial t \partial z}\left(\frac{\partial^{2} w}{\partial x^{2}}-\frac{\partial^{2} u}{\partial x \partial z}\right)$

$+U_{0} \frac{\partial^{3}}{\partial t \partial x^{2}}\left(\frac{\partial w}{\partial x}-\frac{\partial u}{\partial z}\right)+\left(u+U_{0}\right) U_{0} \frac{\partial^{3}}{\partial x^{3}}\left(\frac{\partial w}{\partial x}-\frac{\partial u}{\partial z}\right)+$

$\left(v+V_{0}\right) U_{0} \frac{\partial^{3}}{\partial x^{2} \partial y}\left(\frac{\partial w}{\partial x}-\frac{\partial u}{\partial z}\right)+w U_{0} \frac{\partial^{3}}{\partial x^{2} \partial z}\left(\frac{\partial w}{\partial x}-\frac{\partial u}{\partial z}\right)$

$+V_{0} \frac{\partial^{3}}{\partial t \partial x \partial y}\left(\frac{\partial w}{\partial x}-\frac{\partial u}{\partial z}\right)+\left(u+U_{0}\right) V_{0} \frac{\partial^{3}}{\partial x^{2} \partial y}\left(\frac{\partial w}{\partial x}-\frac{\partial u}{\partial z}\right)$

$+\left(v+V_{0}\right) V_{0} \frac{\partial^{3}}{\partial x \partial y^{2}}\left(\frac{\partial w}{\partial x}-\frac{\partial u}{\partial z}\right)+w V_{0} \frac{\partial^{3}}{\partial x \partial y \partial z}\left(\frac{\partial w}{\partial x}-\frac{\partial u}{\partial z}\right)$

$-\frac{\partial}{\partial t}\left(\frac{\partial U_{0}}{\partial z} \frac{\partial^{2} u}{\partial x^{2}}+\frac{\partial V_{0}}{\partial z} \frac{\partial^{2} u}{\partial x \partial y}\right)-\left(u+U_{0}\right) \frac{\partial}{\partial x}\left(\frac{\partial U_{0}}{\partial z} \frac{\partial^{2} u}{\partial x^{2}}+\right.$

$\left.\frac{\partial V_{0}}{\partial z} \frac{\partial^{2} u}{\partial x \partial y}\right)-\left(v+V_{0}\right) \frac{\partial}{\partial y}\left(\frac{\partial U_{0}}{\partial z} \frac{\partial^{2} u}{\partial x^{2}}+\frac{\partial V_{0}}{\partial z} \frac{\partial^{2} u}{\partial x \partial y}\right)-$

$w \frac{\partial}{\partial z}\left(\frac{\partial U_{0}}{\partial z} \frac{\partial^{2} u}{\partial x^{2}}+\frac{\partial V_{0}}{\partial z} \frac{\partial^{2} u}{\partial x \partial y}\right)-\frac{\partial}{\partial t} \frac{\partial^{2}}{\partial x \partial z}\left(w \frac{\partial U_{0}}{\partial z}\right)-$ 


$$
\begin{aligned}
& \left(u+U_{0}\right) \frac{\partial}{\partial x} \frac{\partial^{2}}{\partial x \partial z}\left(w \frac{\partial U_{0}}{\partial z}\right)-\left(v+V_{0}\right) \frac{\partial^{3}}{\partial x \partial y \partial z}\left(w \frac{\partial U_{0}}{\partial z}\right) \\
& -w \frac{\partial^{3}}{\partial x \partial z^{2}}\left(w \frac{\partial U_{0}}{\partial z}\right)+f \frac{\partial^{3} v}{\partial t \partial x \partial z}+f\left(u+U_{0}\right) \frac{\partial^{2} v}{\partial x^{2} \partial z} \\
& +f\left(v+V_{0}\right) \frac{\partial^{3} v}{\partial x \partial y \partial z}+f w \frac{\partial^{3} v}{\partial x \partial z^{2}}- \\
& \tilde{f} \frac{\partial}{\partial t}\left(\frac{\partial^{2} w}{\partial x \partial z}+\frac{\partial^{2} u}{\partial x^{2}}\right)-\tilde{f}\left(u+U_{0}\right) \frac{\partial}{\partial x}\left(\frac{\partial^{2} w}{\partial x \partial z}+\frac{\partial^{2} u}{\partial x^{2}}\right)- \\
& \tilde{f}\left(v+V_{0}\right) \frac{\partial}{\partial y}\left(\frac{\partial^{2} w}{\partial x \partial z}+\frac{\partial^{2} u}{\partial x^{2}}\right)-\tilde{f} w \frac{\partial}{\partial z}\left(\frac{\partial^{2} w}{\partial x \partial z}+\frac{\partial^{2} u}{\partial x^{2}}\right) \\
& =\frac{\partial^{3} b}{\partial t \partial x^{2}}+\left(u+U_{0}\right) \frac{\partial^{3} b}{\partial x^{3}}+\left(v+V_{0}\right) \frac{\partial^{3} b}{\partial x \partial y^{2}}+w \frac{\partial^{3} b}{\partial x^{2} \partial z}
\end{aligned}
$$

The derivation process is similar to (8)--(12), and we derive Equation (2) with respect to the variable $z$, then:

$$
\begin{aligned}
& \frac{\partial^{2} v}{\partial t \partial z}+\frac{\partial w}{\partial z} \frac{\partial V_{0}}{\partial z}+w \frac{\partial^{2} V_{0}}{\partial z^{2}}+\frac{\partial U_{0}}{\partial z} \frac{\partial v}{\partial x}+ \\
& U_{0} \frac{\partial^{2} v}{\partial x \partial z}+\frac{\partial V_{0}}{\partial z} \frac{\partial v}{\partial y}+V_{0} \frac{\partial^{2} v}{\partial y \partial z}+f\left(\frac{\partial u}{\partial z}\right. \\
& \left.+\frac{\partial U_{0}}{\partial z}\right)=-\frac{\partial^{2} p}{\partial y \partial z}
\end{aligned}
$$

Then the derivative of Equation (3) is with respect to the variable $y$, so:

$$
\begin{aligned}
& \left(\frac{\partial^{2} w}{\partial t \partial y}+U_{0} \frac{\partial^{2} w}{\partial x \partial y}+V_{0} \frac{\partial^{2} w}{\partial y^{2}}\right)-\tilde{f} \frac{\partial u}{\partial y}, \\
& =-\frac{\partial^{2} p}{\partial y \partial z}+\frac{\partial b}{\partial y}
\end{aligned}
$$

Subtract Equation (13) from Equation (14), and then we obtain:

$$
\begin{aligned}
& \frac{\partial}{\partial t}\left(\frac{\partial w}{\partial y}-\frac{\partial v}{\partial z}\right)+U_{0} \frac{\partial}{\partial x}\left(\frac{\partial w}{\partial y}-\frac{\partial v}{\partial z}\right)+V_{0} \frac{\partial}{\partial y}\left(\frac{\partial w}{\partial y}-\frac{\partial v}{\partial z}\right)(15) \\
& -\frac{\partial}{\partial z}\left(w \frac{\partial V_{0}}{\partial z}\right)-\left(\frac{\partial U_{0}}{\partial z} \frac{\partial v}{\partial x}+\frac{\partial V_{0}}{\partial z} \frac{\partial v}{\partial y}\right)-f\left(\frac{\partial u}{\partial z}+\frac{\partial U_{0}}{\partial z}\right) \\
& -\tilde{f} \frac{\partial u}{\partial y}=\frac{\partial b}{\partial y}
\end{aligned}
$$

Then derivative of Equation (15) is with respect to the variable ${ }^{y}$, so:

$\frac{\partial}{\partial t}\left(\frac{\partial^{2} w}{\partial y^{2}}-\frac{\partial^{2} v}{\partial y \partial z}\right)+U_{0} \frac{\partial}{\partial x}\left(\frac{\partial^{2} w}{\partial y^{2}}-\frac{\partial^{2} v}{\partial y \partial z}\right)$

$+V_{0} \frac{\partial}{\partial y}\left(\frac{\partial^{2} w}{\partial y^{2}}-\frac{\partial v}{\partial y \partial z}\right)-\frac{\partial^{2}}{\partial y \partial z}\left(w \frac{\partial V_{0}}{\partial z}\right)-$

$\frac{\partial}{\partial y}\left(\frac{\partial U_{0}}{\partial z} \frac{\partial v}{\partial x}+\frac{\partial V_{0}}{\partial z} \frac{\partial v}{\partial y}\right)-f \frac{\partial^{2} u}{\partial y \partial z}$

$-\tilde{f} \frac{\partial^{2} u}{\partial y^{2}}=\frac{\partial^{2} b}{\partial y^{2}}$

And then the linear derivative operator is used in
Equation (16), which can be:

$$
\begin{aligned}
& \frac{\partial^{2}}{\partial t^{2}}\left(\frac{\partial^{2} w}{\partial y^{2}}-\frac{\partial^{2} v}{\partial y \partial z}\right)+\left(u+U_{0}\right) \frac{\partial^{2}}{\partial t \partial x}\left(\frac{\partial^{2} w}{\partial y^{2}}\right. \\
& \left.-\frac{\partial^{2} v}{\partial y \partial z}\right)+\left(v+V_{0}\right) \frac{\partial^{2}}{\partial t \partial y}\left(\frac{\partial^{2} w}{\partial y^{2}}-\frac{\partial^{2} v}{\partial y \partial z}\right)+ \\
& w \frac{\partial^{2}}{\partial t \partial z}\left(\frac{\partial^{2} w}{\partial y^{2}}-\frac{\partial^{2} v}{\partial y \partial z}\right)+U_{0} \frac{\partial^{2}}{\partial t \partial x}\left(\frac{\partial^{2} w}{\partial y^{2}}-\right. \\
& \left.\frac{\partial^{2} v}{\partial y \partial z}\right)+\left(u+U_{0}\right) U_{0} \frac{\partial^{2}}{\partial x^{2}}\left(\frac{\partial^{2} w}{\partial y^{2}}-\frac{\partial^{2} v}{\partial y \partial z}\right) \\
& +\left(v+V_{0}\right) U_{0} \frac{\partial^{2}}{\partial x \partial y}\left(\frac{\partial^{2} w}{\partial y^{2}}-\frac{\partial^{2} v}{\partial y \partial z}\right)+ \\
& U_{0} w \frac{\partial^{2}}{\partial x \partial z}\left(\frac{\partial^{2} w}{\partial y^{2}}-\frac{\partial^{2} v}{\partial y \partial z}\right)+V_{0} \frac{\partial^{2}}{\partial t \partial y}\left(\frac{\partial^{2} w}{\partial y^{2}}\right. \\
& \left.-\frac{\partial v}{\partial y \partial z}\right)+\left(u+U_{0}\right) V_{0} \frac{\partial^{2}}{\partial x \partial y}\left(\frac{\partial^{2} w}{\partial y^{2}}-\frac{\partial v}{\partial y \partial z}\right) \\
& +\left(v+V_{0}\right) V_{0} \frac{\partial^{2}}{\partial y^{2}}\left(\frac{\partial^{2} w}{\partial y^{2}}-\frac{\partial v}{\partial y \partial z}\right)+ \\
& V_{0} w \frac{\partial^{2}}{\partial y \partial z}\left(\frac{\partial^{2} w}{\partial y^{2}}-\frac{\partial v}{\partial y \partial z}\right)-\frac{\partial^{3}}{\partial t \partial y \partial z}\left(w \frac{\partial V_{0}}{\partial z}\right) \\
& -\left(u+U_{0}\right) \frac{\partial^{3}}{\partial x \partial y \partial z}\left(w \frac{\partial V_{0}}{\partial z}\right)- \\
& \left(v+V_{0}\right) \frac{\partial^{3}}{\partial y^{2} \partial z}\left(w \frac{\partial V_{0}}{\partial z}\right)-w \frac{\partial^{3}}{\partial y \partial z^{2}}\left(w \frac{\partial V_{0}}{\partial z}\right) \\
& -\frac{\partial^{2}}{\partial t \partial y}\left(\frac{\partial U_{0}}{\partial z} \frac{\partial v}{\partial x}+\frac{\partial V_{0}}{\partial z} \frac{\partial v}{\partial y}\right)- \\
& \left(u+U_{0}\right) \frac{\partial^{2}}{\partial x \partial y}\left(\frac{\partial U_{0}}{\partial z} \frac{\partial v}{\partial x}+\frac{\partial V_{0}}{\partial z} \frac{\partial v}{\partial y}\right) \\
& -\left(v+V_{0}\right) \frac{\partial^{2}}{\partial y^{2}}\left(\frac{\partial U_{0}}{\partial z} \frac{\partial v}{\partial x}+\frac{\partial V_{0}}{\partial z} \frac{\partial v}{\partial y}\right) \\
& -w \frac{\partial^{2}}{\partial y \partial z}\left(\frac{\partial U_{0}}{\partial z} \frac{\partial v}{\partial x}+\frac{\partial V_{0}}{\partial z} \frac{\partial v}{\partial y}\right) \\
& -f \frac{\partial^{3} u}{\partial t \partial y \partial z}-f\left(u+U_{0}\right) \frac{\partial^{3} u}{\partial x \partial y \partial z} \\
& -f\left(v+V_{0}\right) \frac{\partial^{3} u}{\partial y^{2} \partial z}-f w \frac{\partial^{3} u}{\partial y \partial z^{2}} \\
& -\tilde{f} \frac{\partial^{3} u}{\partial t \partial y^{2}}-\tilde{f}\left(u+U_{0}\right) \frac{\partial^{3} u}{\partial x \partial y^{2}} \\
& -\tilde{f}\left(v+V_{0}\right) \frac{\partial^{3} u}{\partial y^{3}}-\tilde{f} w \frac{\partial^{3} u}{\partial y^{2} \partial z} \\
& =\frac{\partial^{3} b}{\partial t \partial y^{2}}+\left(u+U_{0}\right) \frac{\partial^{3} b}{\partial x \partial y^{2}} \\
& +\left(v+V_{0}\right) \frac{\partial^{3} b}{\partial y^{3}}+w \frac{\partial^{3} b}{\partial y^{2} \partial z}
\end{aligned}
$$




\section{MATEC Web of Conferences}

Add Equation (12) and Equation (17), and put the result in order, then:

$\left(\frac{\partial}{\partial t}+\left(u+U_{0}\right) \frac{\partial}{\partial x}+\left(v+V_{0}\right) \frac{\partial}{\partial y}+w \frac{\partial}{\partial z}\right) \frac{\partial}{\partial t}\left(\frac{\partial^{2} w}{\partial x^{2}}+\frac{\partial^{2} w}{\partial y^{2}}-\frac{\partial}{\partial z}\left(\frac{\partial u}{\partial x}+\frac{\partial v}{\partial y}\right)\right)$
$+U_{0}\left(\frac{\partial}{\partial t}+\left(u+U_{0}\right) \frac{\partial}{\partial x}+\left(v+V_{0}\right) \frac{\partial}{\partial y}+w \frac{\partial}{\partial z}\right) \frac{\partial}{\partial x}\left(\frac{\partial^{2} w}{\partial x^{2}}+\frac{\partial^{2} w}{\partial y^{2}}-\frac{\partial}{\partial z}\left(\frac{\partial u}{\partial x}+\frac{\partial v}{\partial y}\right)\right)$
$+V_{0}\left(\frac{\partial}{\partial t}+\left(u+U_{0}\right)_{0} \frac{\partial}{\partial x}+\left(v+V_{0}\right) \frac{\partial}{\partial y}+w \frac{\partial}{\partial z}\right) \frac{\partial}{\partial y}\left(\frac{\partial^{2} w}{\partial x^{2}}+\frac{\partial^{2} w}{\partial y^{2}}-\frac{\partial}{\partial z}\left(\frac{\partial u}{\partial x}+\frac{\partial v}{\partial y}\right)\right)$
$-\left(\frac{\partial}{\partial t}+\left(u+U_{0}\right) \frac{\partial}{\partial x}+\left(v+V_{0}\right) \frac{\partial}{\partial y}+w \frac{\partial}{\partial z}\right)\left(\frac{\partial U_{0}}{\partial z} \frac{\partial}{\partial x}+\frac{\partial V_{0}}{\partial z} \frac{\partial}{\partial y}\right)\left(\frac{\partial u}{\partial x}+\frac{\partial v}{\partial y}\right)$
$-\left(\frac{\partial^{2}}{\partial t}+\left(u+U_{0}\right) \frac{\partial}{\partial x}+\left(v+V_{0}\right) \frac{\partial}{\partial y}+w \frac{\partial}{\partial z}\right) \frac{\partial}{\partial z}\left(\frac{\partial U_{0}}{\partial z} \frac{\partial w}{\partial x}+\frac{\partial V_{0}}{\partial z} \frac{\partial w}{\partial y}\right)$
$-f\left(\frac{\partial}{\partial t}+\left(u+U_{0}\right) \frac{\partial}{\partial x}+\left(v+V_{0}\right) \frac{\partial}{\partial y}+w \frac{\partial}{\partial z}\right) \frac{\partial}{\partial z}\left(\frac{\partial u}{\partial y}-\frac{\partial v}{\partial x}\right)$
$-\tilde{f}\left(\frac{\partial}{\partial t}+\left(u+U_{0}\right) \frac{\partial}{\partial x}+\left(v+V_{0}\right) \frac{\partial}{\partial y}+w \frac{\partial}{\partial z}\right)\left(\frac{\partial^{2} u}{\partial x^{2}}+\frac{\partial^{2} u}{\partial y^{2}}\right)$
$-\tilde{f}\left(\frac{\partial}{\partial t}+\left(u+U_{0}\right) \frac{\partial}{\partial x}+\left(v+V_{0}\right) \frac{\partial}{\partial y}+w \frac{\partial}{\partial z}\right) \frac{\partial^{2} w}{\partial x \partial z}$
$=\left(\frac{\partial}{\partial t}+\left(u+U_{0}\right) \frac{\partial}{\partial x}+\left(v+V_{0}\right) \frac{\partial}{\partial y}+w \frac{\partial}{\partial z}\right)\left(\frac{\partial^{2} b}{\partial x^{2}}+\frac{\partial^{2} b}{\partial y^{2}}\right)$

Which is:

$\frac{d}{d t}\left[\frac{D}{D t}\left(\frac{\partial^{2} w}{\partial x^{2}}+\frac{\partial^{2} w}{\partial y^{2}}+\frac{\partial^{2} w}{\partial z^{2}}\right)\right.$

$-\left(\frac{\partial U_{0}}{\partial z} \frac{\partial}{\partial x}+\frac{\partial V_{0}}{\partial z} \frac{\partial}{\partial y}\right)\left(\frac{\partial u}{\partial x}+\frac{\partial v}{\partial y}\right)$

$-\frac{\partial}{\partial z}\left(\frac{\partial U_{0}}{\partial z} \frac{\partial w}{\partial x}+\frac{\partial V_{0}}{\partial z} \frac{\partial w}{\partial y}\right)$

$-\tilde{f}\left(\frac{\partial^{2} u}{\partial x^{2}}+\frac{\partial^{2} u}{\partial y^{2}}+\frac{\partial^{2} w}{\partial x \partial z}\right)$

$\left.-f \frac{\partial}{\partial z}\left(\frac{\partial u}{\partial y}-\frac{\partial v}{\partial x}\right)\right]=\frac{d}{d t}\left(\frac{\partial^{2} b}{\partial x^{2}}+\frac{\partial^{2} b}{\partial y^{2}}\right)$

Derive Equation (1) with respect to the variable $\mathcal{y}$, then:

$\frac{\partial^{2} u}{\partial t \partial y}+\frac{\partial U_{0}}{\partial z} \frac{\partial w}{\partial y}+U_{0} \frac{\partial^{2} u}{\partial x \partial y}+V_{0} \frac{\partial^{2} u}{\partial y^{2}}$

$-f \frac{\partial v}{\partial y}+\tilde{f} \frac{\partial w}{\partial y}=-\frac{\partial^{2} p}{\partial x \partial y}$

$\frac{\partial}{\partial t}\left(\frac{\partial u}{\partial y}-\frac{\partial v}{\partial x}\right)+\frac{\partial V_{0}}{\partial z}\left(\frac{\partial u}{\partial y}-\frac{\partial v}{\partial x}\right)+$

$U_{0} \frac{\partial}{\partial x}\left(\frac{\partial u}{\partial y}-\frac{\partial v}{\partial x}\right)+V_{0} \frac{\partial}{\partial y}\left(\frac{\partial u}{\partial y}-\frac{\partial v}{\partial x}\right)$

$-f\left(\frac{\partial u}{\partial x}+\frac{\partial v}{\partial y}\right)+\tilde{f} \frac{\partial w}{\partial y}=0$

Take the Continuity Equation (5) into Equation (21), so:

$\left(\frac{\partial}{\partial t}+U_{0} \frac{\partial}{\partial x}+V_{0} \frac{\partial}{\partial y}\right)\left(\frac{\partial u}{\partial y}-\frac{\partial v}{\partial x}\right)$

$=\frac{\partial V_{0}}{\partial z} \frac{\partial w}{\partial x}-\frac{\partial U_{0}}{\partial z} \frac{\partial w}{\partial y}-f \frac{\partial w}{\partial z}-\tilde{f} \frac{\partial w}{\partial y}$

Then derive Equation (5):

$\frac{\partial u}{\partial x}+\frac{\partial v}{\partial y}=-\frac{\partial w}{\partial z}$

With the derivative operator:

$\frac{D}{D t}=\frac{\partial}{\partial t}+U_{0} \frac{\partial}{\partial x}+V_{0} \frac{\partial}{\partial y}$

We obtain:

$\frac{D}{D t}\left(\frac{\partial u}{\partial x}+\frac{\partial v}{\partial y}\right)=-\frac{\partial^{2} w}{\partial t \partial z}-U_{0} \frac{\partial^{2} w}{\partial x \partial z}-V_{0} \frac{\partial^{2} w}{\partial y \partial z},(25)$

The derivative of Equation (22) is with respect to the variable $\mathcal{Y}$, then:

$\frac{D}{D t}\left(\frac{\partial^{2} u}{\partial y^{2}}-\frac{\partial^{2} v}{\partial x \partial y}\right)=\frac{\partial V_{0}}{\partial z} \frac{\partial^{2} w}{\partial x \partial y}-\frac{\partial U_{0}}{\partial z} \frac{\partial^{2} w}{\partial y^{2}}$

$-f \frac{\partial^{2} w}{\partial y \partial z}-\tilde{f} \frac{\partial^{2} w}{\partial y^{2}}$

And the derivative of Equation (25) is with respect to the variable $X$, so:

$\frac{D}{D t}\left(\frac{\partial^{2} u}{\partial x^{2}}+\frac{\partial^{2} v}{\partial x \partial y}\right)=-\frac{\partial^{3} w}{\partial t \partial x \partial z}-U_{0} \frac{\partial^{3} w}{\partial x^{2} \partial z}-V_{0} \frac{\partial^{3} w}{\partial x \partial y \partial z}$

Let Equation (27) add Equation (26), then we can get:

$\frac{D}{D t}\left(\frac{\partial^{2} u}{\partial x^{2}}+\frac{\partial^{2} u}{\partial y^{2}}\right)=\frac{\partial V_{0}}{\partial z} \frac{\partial^{2} w}{\partial x \partial y}-\frac{\partial U_{0}}{\partial z} \frac{\partial^{2} w}{\partial y^{2}}$

Then the derivative of Equation (2) is with respect to the variable $x$, so:

$\frac{\partial^{2} v}{\partial t \partial x}+\frac{\partial V_{0}}{\partial z} \frac{\partial w}{\partial x}+U_{0} \frac{\partial^{2} v}{\partial x^{2}}+V_{0} \frac{\partial^{2} v}{\partial x \partial y}$

$+f \frac{\partial u}{\partial x}=-\frac{\partial^{2} p}{\partial x \partial y}$

$-f \frac{\partial^{2} w}{\partial y \partial z}-\tilde{f} \frac{\partial^{2} w}{\partial y^{2}}-\frac{\partial^{3} w}{\partial t \partial x \partial z}-U_{0} \frac{\partial^{3} w}{\partial x^{2} \partial z}-V_{0} \frac{\partial^{3} w}{\partial x \partial y \partial z}$

From Equation (22), we can get:

$\frac{\partial^{3} b}{\partial t \partial x^{2}}+U_{0} \frac{\partial^{3} b}{\partial x^{3}}+V_{0} \frac{\partial^{3} b}{\partial x^{2} \partial y}+N^{2} \frac{\partial^{2} w}{\partial x^{2}}=0$

Subtract Equation (19) from Equation (20), and then we obtain:

And:

$\frac{\partial^{3} b}{\partial t \partial y^{2}}+U_{0} \frac{\partial^{3} b}{\partial x \partial y^{2}}+V_{0} \frac{\partial^{3} b}{\partial y^{3}}+N^{2} \frac{\partial^{2} w}{\partial y^{2}}=0$ 
Then:

$\frac{D}{D t}\left(\frac{\partial^{2} b}{\partial x^{2}}+\frac{\partial^{2} b}{\partial y^{2}}\right)=-N^{2}\left(\frac{\partial^{2} w}{\partial x^{2}}+\frac{\partial^{2} w}{\partial y^{2}}\right)$

Take Equations (22), (23), (28) and (31) into Equation (18), the vertical velocity equation of the fluid movement is as follows:

$$
\begin{aligned}
& \frac{d}{d t}\left\{\frac{D^{2}}{D t^{2}}\left(\frac{\partial^{2} w}{\partial x^{2}}+\frac{\partial^{2} w}{\partial y^{2}}+\frac{\partial^{2} w}{\partial z^{2}}\right)\right. \\
& +\frac{D}{D t}\left[\left(\frac{\partial U_{0}}{\partial z} \frac{\partial}{\partial x}+\frac{\partial V_{0}}{\partial z} \frac{\partial}{\partial y}\right) \frac{\partial w}{\partial z}\right] \\
& -\frac{D}{D t}\left[\frac{\partial}{\partial z}\left(\frac{\partial U_{0}}{\partial z} \frac{\partial w}{\partial x}+\frac{\partial V_{0}}{\partial z} \frac{\partial w}{\partial y}\right)\right] \\
& -f\left(\frac{\partial}{\partial z} \frac{\partial V_{0}}{\partial z} \frac{\partial w}{\partial x}-\frac{\partial U_{0}}{\partial z} \frac{\partial w}{\partial y}-f \frac{\partial w}{\partial z}\right. \\
& \left.-\tilde{f} \frac{\partial w}{\partial y}\right)-\tilde{f}\left(\frac{\partial V_{0}}{\partial z} \frac{\partial^{2} w}{\partial x \partial y}-\frac{\partial U_{0}}{\partial z} \frac{\partial^{2} w}{\partial y^{2}}\right. \\
& -f \frac{\partial^{2} w}{\partial y \partial z}-\tilde{f} \frac{\partial^{2} w}{\partial y^{2}}-\frac{\partial^{3} w}{\partial t \partial x \partial z}- \\
& \left.U_{0} \frac{\partial^{3} w}{\partial x^{2} \partial z}-V_{0} \frac{\partial^{3} w}{\partial x \partial y \partial z}\right)-\tilde{f} \frac{D}{D t}\left(\frac{\partial^{2} w}{\partial x \partial z}\right) . \\
& \left.+N^{2}\left(\frac{\partial^{2} w}{\partial x^{2}}+\frac{\partial^{2} w}{\partial y^{2}}\right)\right\}=0
\end{aligned}
$$

The Equation (32) is the vertical velocity equation of the sea water in a horizontal uniform background current field under non-traditional approximation. If the average background flow does not exist, then the Equation (32) will be degenerated to the vertical velocity equation of internal-wave. Equation (32) and boundary conditions (6) and (7) can determine the vertical velocity of fluid motion in a horizontal uniform steady current field, and other wave field elements can be expressed by the vertical velocity $w$ as follows:

$$
\begin{aligned}
& \frac{D}{D t}\left(\frac{\partial^{2} u}{\partial x^{2}}+\frac{\partial^{2} u}{\partial y^{2}}\right) \\
& =\frac{\partial V_{0}}{\partial z} \frac{\partial^{2} w}{\partial x \partial y}-\frac{\partial U_{0}}{\partial z} \frac{\partial^{2} w}{\partial y^{2}}-, \\
& f \frac{\partial^{2} w}{\partial y \partial z}-\tilde{f} \frac{\partial^{2} w}{\partial y^{2}}-\frac{\partial^{3} w}{\partial t \partial x \partial z} \\
& -U_{0} \frac{\partial^{3} w}{\partial x^{2} \partial z}-V_{0} \frac{\partial^{3} w}{\partial x \partial y \partial z} \\
& \text { And: }
\end{aligned}
$$

$$
\begin{aligned}
& \frac{D}{D t}\left(\frac{\partial^{2} v}{\partial x^{2}}+\frac{\partial^{2} v}{\partial y^{2}}\right) \\
& =-\frac{D}{D t}\left(\frac{\partial w}{\partial y \partial z}\right)-\frac{\partial V_{0}}{\partial z} \frac{\partial^{2} w}{\partial x^{2}} \\
& +\frac{\partial U_{0}}{\partial z} \frac{\partial^{2} w}{\partial x \partial y}+f \frac{\partial^{2} w}{\partial x \partial z}+\tilde{f} \frac{\partial^{2} w}{\partial x \partial y} \\
& \frac{d \zeta}{d t}=w
\end{aligned}
$$

\subsection{Wave solution and dispersion relation}

We assume that the vertical velocity has the form:

$$
w(x, y, z, t)=W(z) \exp i(k x+l y-\omega t)
$$

Where, $k$ and $l$ are the wavenumbers in $x$ and $y$. By substituting (36) into (32) and eliminating the exponential factor, we can obtain the following equations for $W(\mathrm{z})$ :

$$
\begin{aligned}
& {\left[f^{2}-\left(U_{0} k+V_{0} l-\omega\right)^{2}\right] W^{\prime \prime}(z)+} \\
& +2 i l i \tilde{f} W^{\prime}(z)+ \\
& \left\{\left[-N^{2}+\left(U_{0} k+V_{0} l-\omega\right)^{2}\right]\left(k^{2}+l^{2}\right)-\tilde{f}^{2} l^{2}\right. \\
& \left.+\left(\frac{\partial^{2} U_{0}}{\partial z^{2}} k+\frac{\partial^{2} V_{0}}{\partial z^{2}} l\right)\left(-\omega+U_{0} k+V_{0} l\right)\right\} W(z)=0
\end{aligned}
$$

Equation (37) and boundary conditions (6)--(7) formed the vertical velocity equation of fluid motion in a horizontal uniform current field. Let:

$$
W(z)=\Phi(z) \mathrm{e}^{i \gamma z}
$$

Where, $\gamma$ is a real coefficient that will be determined later. By substituting (38) into (23) and (37), we can obtain the following equation:

$$
\begin{aligned}
& {\left[f^{2}-\left(U_{0} k+V_{0} l-\omega\right)^{2}\right] \Phi^{\prime \prime}(z)} \\
& +\left\{2 i \gamma\left[f^{2}-\left(U_{0} k+V_{0} l-\omega\right)^{2}\right]+2 i l \tilde{f} f\right\} \Phi^{\prime}(z) \\
& +\left\{-\gamma^{2}\left(f^{2}-\left(U_{0} k+V_{0} l-\omega\right)^{2}\right)-2 \gamma l \tilde{f} \bar{f}\right. \\
& +\left[-N^{2}+\left(U_{0} k+V_{0} l-\omega\right)^{2}\right]\left(k^{2}+l^{2}\right)-\tilde{f}^{2} l^{2}+ \\
& \left.\left(\frac{\partial^{2} U_{0}}{\partial z^{2}} k+\frac{\partial^{2} V_{0}}{\partial z^{2}} l\right)\left(\omega-U_{0} k-V_{0} l\right)\right\} \Phi(z)=0
\end{aligned}
$$

Let the coefficient of $\Phi^{\prime}(z)$ equals 0 , then: 


\section{MATEC Web of Conferences}

$\gamma=\frac{-l \tilde{f}}{f^{2}-\left(U_{0} k+V_{0} l-\omega\right)^{2}}$

The Equation (39) is changed into the following form:

$$
\begin{aligned}
& \Phi^{\prime \prime}(z) \\
& +\left\{\frac{\left[-N^{2}+\left(U_{0} k+V_{0} l-\omega\right)^{2}\right]\left(k^{2}+l^{2}\right)-\tilde{f}^{2} l^{2}}{f^{2}-\left(U_{0} k+V_{0} l-\omega\right)^{2}}\right. \\
& +\left(\frac{\partial^{2} U_{0}}{\partial z^{2}} k+\frac{\partial^{2} V_{0}}{\partial z^{2}} l\right) \frac{\left(\omega-U_{0} k-V_{0} l\right)}{f^{2}-\left(U_{0} k+V_{0} l-\omega\right)^{2}} \\
& \left.+\frac{(l \tilde{f})^{2}}{\left[f^{2}-\left(U_{0} k+V_{0} l-\omega\right)^{2}\right]^{2}}\right\} \Phi(z)=0
\end{aligned}
$$

When not considering the effect of the background current field, (41) reduced to the equations is the Boussinesq version of the similar equations ( 7 on page 82 of Liu Yongjun ${ }^{[11]}$.

Our task now is to solve (41) under the boundary conditions (6) and (7) then

\subsection{Solutions of the system in the case of constant $N$}

Because the boundary conditions are $w=0$ at $z=0$ and 1 , the solution of (41) is chosen in the form. The general solutions of the system of Equation (41) can be expressed by:

$$
\Phi(z)=\sin \kappa z
$$

Where, $\kappa$ is the vertical half-wavenumber and it is expressed by:

$$
\begin{aligned}
& \kappa= \\
& \left\{\frac{\left[-N^{2}+\left(U_{0} k+V_{0} l-\omega\right)^{2}\right]\left(k^{2}+l^{2}\right)-\tilde{f}^{2} l^{2}}{f^{2}-\left(U_{0} k+V_{0} l-\omega\right)^{2}}\right. \\
& +\left(\frac{\partial^{2} U_{0}}{\partial z^{2}} k+\frac{\partial^{2} V_{0}}{\partial z^{2}} l\right) \frac{\left(\omega-U_{0} k-V_{0} l\right)}{f^{2}-\left(U_{0} k+V_{0} l-\omega\right)^{2}} \\
& +\frac{(l f \tilde{f})^{2}}{\left[f^{2}-\left(U_{0} k+V_{0} l-\omega\right)^{2}\right]^{2}}=\frac{j \pi}{H}
\end{aligned}
$$

And $j=1,2,3, \ldots$ respectively denotes the vertical wave mode index. Solution (42) as wave motion satisfies (41), and it provides that:

$$
\begin{aligned}
& \left(\frac{j^{2} \pi^{2}}{H^{2}\left(k^{2}+l^{2}\right)}+1\right) \omega^{4} \\
& -4\left(\frac{j^{2} \pi^{2}}{H^{2}\left(k^{2}+l^{2}\right)}+1\right)\left(U_{0} k+V_{0} l\right) \omega^{3} \\
& +\left(\frac{\partial^{2} U_{0}}{\partial z^{2}} \frac{k}{k^{2}+l^{2}}+\frac{\partial^{2} V_{0}}{\partial z^{2}} \frac{l}{k^{2}+l^{2}}\right) \omega^{3} \\
& +6\left(\frac{j^{2} \pi^{2}}{H^{2}\left(k^{2}+l^{2}\right)}+1\right)\left(U_{0} k+V_{0} l\right)^{2} \omega^{2} \\
& -3\left(\frac{\partial^{2} U_{0}}{\partial z^{2}} \frac{k}{k^{2}+l^{2}}+\frac{\partial^{2} V_{0}}{\partial z^{2}} \frac{l}{k^{2}+l^{2}}\right)\left(U_{0} k+V_{0} l\right) \omega^{2} \\
& -\left(N^{2}+f^{2}+\frac{\tilde{f}^{2} l^{2}}{k^{2}+l^{2}}+\frac{2 f^{2} j^{2} \pi^{2}}{H^{2}\left(k^{2}+l^{2}\right)}\right) \omega^{2} \\
& -4\left(\frac{j^{2} \pi^{2}}{H^{2}\left(k^{2}+l^{2}\right)}+1\right)\left(U_{0} k+V_{0} l\right)^{3} \omega
\end{aligned}
$$

$+3\left(\frac{\partial^{2} U_{0}}{\partial z^{2}} \frac{k}{k^{2}+l^{2}}+\frac{\partial^{2} V_{0}}{\partial z^{2}} \frac{l}{k^{2}+l^{2}}\right)\left(U_{0} k+V_{0} l\right)^{2} \omega$

$+2\left(N^{2}+f^{2}+\frac{\tilde{f}^{2} l^{2}}{k^{2}+l^{2}}+\frac{2 f^{2} j^{2} \pi^{2}}{H^{2}\left(k^{2}+l^{2}\right)}\right)\left(U_{0} k+V_{0} l\right) \omega$

$-\left(\frac{\partial^{2} U_{0}}{\partial z^{2}} \frac{k}{k^{2}+l^{2}}+\frac{\partial^{2} V_{0}}{\partial z^{2}} \frac{l}{k^{2}+l^{2}}\right) f^{2} \omega$

$+\left(\frac{j^{2} \pi^{2}}{H^{2}\left(k^{2}+l^{2}\right)}+1\right)\left(U_{0} k+V_{0} l\right)^{4}$

$-\left(\frac{\partial^{2} U_{0}}{\partial z^{2}} \frac{k}{k^{2}+l^{2}}+\frac{\partial^{2} V_{0}}{\partial z^{2}} \frac{l}{k^{2}+l^{2}}\right)\left(U_{0} k+V_{0} l\right)^{3}$

$-\left(N^{2}+f^{2}+\frac{\tilde{f}^{2} l^{2}}{k^{2}+l^{2}}+\frac{2 f^{2} j^{2} \pi^{2}}{H^{2}\left(k^{2}+l^{2}\right)}\right)\left(U_{0} k+V_{0} l\right)^{2}$

$+\left(\frac{\partial^{2} U_{0}}{\partial z^{2}} \frac{k}{k^{2}+l^{2}}+\frac{\partial^{2} V_{0}}{\partial z^{2}} \frac{l}{k^{2}+l^{2}}\right) f^{2}\left(U_{0} k+V_{0} l\right)$

$+\left(N^{2}+\frac{j^{2} \pi^{2} f^{2}}{H^{2}\left(k^{2}+l^{2}\right)}\right) f^{2}=0$

This is a quartic equation in $\omega$. Therefore, there are four kinds of wave oscillations which propagate in four directions with the different phase speed. The properties of these wave modes are discussed by Kasahara (2003a, 2004), Durran and Bretherton (2004) for the cases of $N=$ constant. Therefore, the readers will refer to those references. However, we should bring out some basics of the dispersion equation (44) that are pertinent to understand the contrast of eigenfunctions between the four different kinds of normal modes. 


\section{CONCLUSIONS}

In this paper, the ocean internal waves dispersion relation under non-traditional approximation derived by Liu Yongjun et al. (2009) is extended to the situation, which the influence of complete Coriolis force and a horizontal uniform background current field on ocean internal waves solution and dispersion relation is considered with the rigid lid approximate boundary conditions of the surface and bottom permeability boundary condition. And we assume that the buoyancy frequency $N$ is constant under the $f$-plane approximation, the internal wave solutions and the dispersion relation are obtained from the linear dynamic equations. Our study shows that there are four kinds of wave oscillations with different phase velocity which propagate in corresponding four directions; it is different from the conclusion of Kasahara and Gary (2006), who confirmed that there are two kinds of wave oscillations which propagate in both eastwards and westwards directions with the same phase speed. Only the effect of complete Coriolis force is considered, but the influence of background current field is not considered.

\section{ACKNOWLEDGEMENT}

The authors (Liu Yongjun et al.) thank Wang Xin for his interest and advice during the course of this research, and thank Kasahara and Gary for their information on the near-inertial oscillations in the oceans. The authors also thank two reviewers' useful comments to increase the readability of this paper. This manuscript was typed by Gao Xiaoping at SWP.

\section{REFERENCES}

[1] M. Ahran. 1990. The North Atlantic Current and Subarctic Intermediate Water. J. Marine Res., 48:-144.

[2] O. Boebel, C. Schmid, \& W. Zenk 1997. Flow and recirculation of Antarctic Intermediate Water across the Rio Grande Rise. J. Geophys. R., 102:967-986.

[3] Kundu, P. K. \& Thomson, R. E. 1985, Inertial Oscillations due to a Moving Front. J. Phys. Oceanogr 15:1076-1084.

[4] Gill, A. E., 1984. On the behaviour of internal waves in the wakes of storms, J. Phys. Oceanogr, 14:1129-1151.

[5] A. Kasahara, A. \& Gary, J.M, 2006, Normal modes of an incompressible and stratified fluid model including the vertical and horizontal components of Coriolis force. Tellus, 58:368-384

[6] Gerkema, T. \& V. I. Shrira. 2005. Near-inertial waves in the ocean: beyond the 'traditional approximation'. $J$ Fluid Mech, 529:195-219.

[7] Garrett, C. and W. 1979. Munk. Internal waves in the ocean. Annu. Rev. Fluid Mech, 11: 339-369

[8] K. Aagaard, A. T. Roach, \& J. D. Schumacher. 1985. On the wind-driven variability of the flow through Bering Strait. Geophys. Res, 90: 7213-7221.

[9] Blumenthal,\& Briscoe, 1995. Distinguishing. propagating waves and standing modes: an internal wave mode 1 . J. Phys. Oceanogr, 25:1095-1115.

[10]Levine, M. D. 2002. A Modification of the Garrett-Munk internal wave spectrum, J. Phys. Oceanogr. 32:166-3181

[11]Liu Yong-jun, Song Jinbao, \& Huang Xiaofeng, 2011. A Kind of WKB Solutions for Internal Waves under the "Non-traditional Approximation", Marine Sciences, 35(1):81-87. 